\title{
Integral Multi-agent Model Recommendation of Learning Objects, for Students and Teachers
}

\author{
Paula Rodríguez, Néstor Duque, and Sara Rodríguez
}

\begin{abstract}
Currently, there has been progress in building models for search and retrieval of learning objects (LO) stored in heterogeneous repositories. Likewise, research has increased the recycling of educational materials. This paper proposes the integration of two multi-agent models focused on delivering, specific LO adapted to a student's profile; and delivering LO to teachers in order to assist them in creating courses. The objective is to have an integral multi-agent model that meets the needs of students and teachers, and in this way improve the teaching learning process.
\end{abstract}

Keywords: Artificial Intelligence in Education, Multi-Agent Systems, Learning Objects, Repositories, Recommendation Systems, e-learning, Virtual organizations, Case Based Reasoning CBR.

\section{Introduction}

The value of information as a learning resource has created the need to share and reuse it without great cost, this added to the development of specifications and standards to solve the problem of incompatibility between different platforms has fueled the emergence of the Learning Object (LO) concept. A LO is a minimum unit of content used to teach something reusable on different platforms. The LO is distinguished from traditional educational resources because they are immediately available in a Web-based repository, to access them through metadata. With the intent of maximizing the number of LO that a user can use in order to support the teaching and learning process, centralized digital repositories are joined in federa-

\author{
Paula Rodríguez · Néstor Duque \\ Universidad Nacional de Colombia \\ e-mail: \{parodriguezma, ndduqueme\} @unal . edu.co \\ Sara Rodríguez \\ Universidad de Salamanca \\ e-mail: srg@usal.es
}


tions of repositories to share and access to other resources [1]. LO must be tagged with metadata, so they can be located and used for educational purposes in Webbased environments [2]. The use of metadata reinforces its utility because of the recovery, localization, exchanges and thus reuse is facilitated. Its importance lies in the fact that through the metadata the initial approach towards the resource can be made and the characteristics can quickly be learned. Recommendation systems are widely used online to support users in finding relevant information [3]. Having a user profile allows the identification of needs and preferences of the student who is doing a search for LO, in order to find the most relevant information or to give recommendations related to which LOs could support the learning process. Intelligent agents are entities that have sufficient autonomy and intelligence to handle specific tasks with little or no human supervision [4]. They are being used almost the same way as traditional systems, making it a good choice for solving problems where one needs autonomous systems to work individually and cooperate with each other to achieve a common goal [5].

The purpose of this paper is to integrate two approaches based on multi-agent systems, that work together to support the teaching and learning processes. The first of which pretend to provide teachers with a tool to recover educational resources to help organize courses [6]; and the second seeks is recommend the most pertinent LO according to the student profile [7]. With the aim of addressing the obstacles that appeared, we propose a model based on multi-agent systems based on virtual organizations that combine the advantages of the two approaches in which we have worked. The rest of the paper is organized as follows: Section 2 outlines main concepts involved in this research; Section 3 describes some works related to the proposed model. Section 4 introduces the multi-agent model proposal. Then, section 5 shows a case study where a course is associated with a teacher, and a student going to the course. LO is recommended from the repository according to the two profiles, a recommendation for a teacher to a student in particular, in order to validate the integration of the two approaches. Finally, conclusions and future work are presented in Section 6.

\section{Basic Concepts}

\subsection{Learning Objects, Repositories and Federations}

Wiley [8] defined LOs as the elements of a new type of instruction based on the object-oriented paradigm (OOP), typical of computer science. The IEEE [9] defines a learning object LO as: any entity, digital or not with instructional design features, capable of being used, reused or referenced during computer-assisted learning. The LO are contained in repositories (LOR) that are specialized digital libraries, they host many types of educational resources and their metadata, which are used in various e-learning environments. There are local repositories defined as digital libraries those specific to an organization that contain further LO own remote repositories that are accessed over a network. The project JORUM [10], 
proposes that a LOR is a collection of LO that has information (metadata) which is detailed and accessible via Internet. Besides housing the LO, the LOR can store the locations of those objects stored elsewhere, both online and in local locations. A federation provides a unified representation of these repositories [11], and serves to facilitate uniform administration of applications to discover and access content in the LO group LOR.

\subsection{Recommender Systems}

Recommendation Systems are aimed to providing users with search results that match their needs, making predictions of their preferences and delivering those items that could come closer than expected [12],[13]. In the context of LOs these systems seek to make recommendations according to the student's characteristics and their learning needs. Furthermore, in order to improve recommendations, recommender systems must perform feedback processes and implement mechanisms that enable them to obtain a large amount of information about users and how they use the LO [1],[14]. On the other hand, to help teachers create their virtual courses, recommender systems should help teachers in the design and creation of courses, suitable for delivering LO educational resources from reuse and sequencing learning activities [15].

The student profile stores information that can be used to obtain search results according to specificity. Managing a user profile aids the student or a teacher in the LO selection according to their preferences and personal characteristics [5].

\subsection{Collaborative Filtering and Case Based Reasoning (CBR)}

The CBR, is a kind of system that solves current problems, using the stored experience that corresponds to past problems. A basic recommendation problem is when a user wants to buy a product; the system recommends products that are related to the first search. If the user accepts the recommendations, this problem is stored. To use a CBR as a recommendation system it is necessary to add 2 to 4 stages of a classic CBR (Retrieve, Reuse, and Retain Check). Moreover, for the definition of this case, there is no predefined model, since it often depends on the problem, but usually includes information about the object, the user, the problem and the solution itself [16].

\subsection{Multi-agent System}

The agents are entities that have the autonomy to perform tasks and achieving their objectives without human supervision. This paradigm presents a new form of analysis, design and implementation of complex software systems and has been used for the development of recommendation systems [5]. 
The desirable characteristics of the agents are as follows [17]: autonomy, reactivity, pro-activity, cooperation and coordination, deliberation, distribution of tasks, adaptation, and parallelism.

\subsection{Virtual Organizations of Agents and Architecture}

The Virtual Organization (VO) is defined as a set of individuals and institutions, which need to coordinate their resources and services within institutional limits [18]. The VO can be considered as open systems formed by grouping and collaboration of heterogeneous entities where there is a clear separation between structure and functionality.

\section{Related Works}

Huang et al. present a framework for the creation of standardized courses, including an e-learning portal LMS, an authoring tool for editing educational resources, a navigation training system, which collects information from the user's learning history and gives course suggestions [19]. However, the two recommendations being executed in this work are not performed. Campos et. al. present a multiagent model to search heterogeneous repositories with semantic features and information users. Besides, a LO classifier is presented which shows user information, statistics and evaluations of objects [20]. In the work of Li et. al. 2012, a ubiquitous learning system is proposed which is based on the student's learning records. This study uses personalized instruction and a context-sensitive method supported by a ubiquitous learning log [21], which only makes recommendations for students.

Casali 2011, presents a recommendation system based on intelligent agents, which aims to return a ranked list of the most suitable LO according to a user profile. The search is performed in the repository Ariadne [5]. The main limitation of this investigation is that despite some student's characteristics are considered in the user profile the user learning styles were not taken into account.

Rodríguez et al. 2012 propose a model for search, retrieval, recommendation and evaluation of LO from repository federation, called BROA, delivering of LO adapted to the student's profile, learning style and preferences, SMA is a studentfocused recommendation [7].

De la Prieta et al. 2011, present BRENHET2 search of educational resources in heterogeneous, multi-agent systems to facilitate reuse of LO through federated search that incorporates a phase of cataloging and filtering results. Federated search is based on the application of virtual organizations SMA [6].

The last two models covering different approaches and looks promising them together to have comprehensive system. 


\section{Proposed Model}

We propose a multi-agent system for search, retrieval, and recommendation of LO, for students and teachers. The LO results of the search, for students are recommended to the profile and learning style. For teachers the system facilitates the composition of training courses from cataloging LO. The search is performed in local and remote repositories, or federations of repositories, accessible via web and descriptive metadata of these objects. It keeps the MAS approach in order to exploit their advantages, among which are: parallelism, the ability of deliberation, Cooperation, Coordination and Distribution. In addition to the federated search process, the MAS has rules and social organizations due to the high heterogeneity of this context. Figure 1 shows the system architecture.

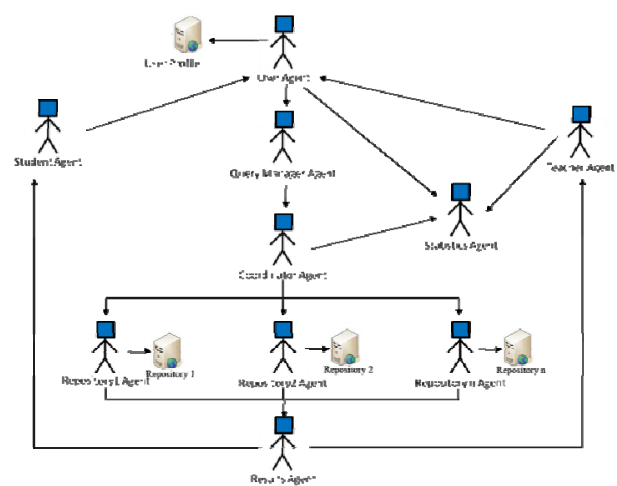

Fig. 1 System Architecture

To make the student's recommendation, using the student profile that consists of user information and preferences, and learning style; LO is recovered from the LOR and the search is performed by metadata title, description and keywords, to find the similarity with the preferences also a match is made between learning style and metadata: learning Resource Type, Interactivity Level, Intended End User Role, Definition, Description and LanguageSystem Architecture.

\subsection{Agent Description}

User Agent: Represents the human user into the system. It has the functions of starting the search process, validating results and also has access to statistics. It manages the user's profile allowing the creation and modification of the characteristics and preferences according to the profile. Query Manager Agent: Is the responsible of monitoring the entire search process. It has the functions to retrieve the query, start the search process, cataloging request and retrieve results from the 
query statistics. Coordinator Agent: This agent redirects the user queries to the search repositories. It has the specific control over the process specific federated search. Also, it should monitor the correct operation of these agents to maximize performance. Repository Agent: Repository agents are responsible of making the actual searches in the repositories. It implements the possible middleware code layers. There will be as many LOR agents, as repositories, which are directed toward the query. Results Agent: Receives the LO from each LOR agent. Automatically extracts metadata schema information and removes invalid LO, and finally calculates a reuse statistic. Teacher Agent: Is responsible for developing the ranking of results. It implements a CBR to develop a ranking of the LO will best suit the needs of the teacher on the basis of information previously obtained. Student Agent: Recommendations to students are made from the LO after user login query. This recommendation is based on the student's learning style. Statistics Agent: This agent is responsible of collecting statistical data from other agents (ROA, evaluation of results and LO). It communicates with the local LOR agent to store these evaluations; the relationship remains LO - evaluation - user.

\section{Experiments and Results}

To evaluate the proposal was made searches with MAS and crossed the recommendations made by the teacher agent and the student agent that would be valid for an integrated system. Multiple searches were performed with the two models, one of which is shown in Figure 2, the query string was cardiology, and Figure 2 shows the recommended LO system of teachers and students; on the right it shows the $\mathrm{LO}$ recommended by the proposed system.

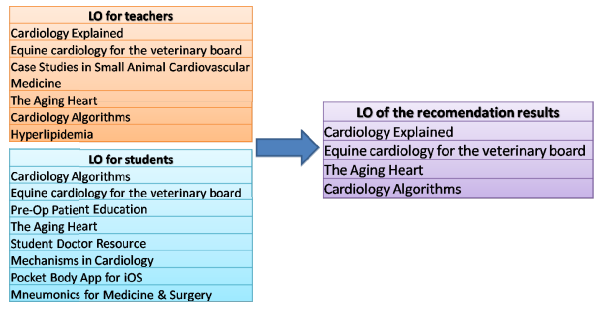

Fig. 2 Result LO list of the three recommendation system

Several tests were performed at 2 systems; the teachers and students with the same query string, as was done with the system proposed in this paper, the amount of recovered LO is shown in the Figure 3. With the proposed system are selected LO that are relevant to a course of a teacher where one student attends. 


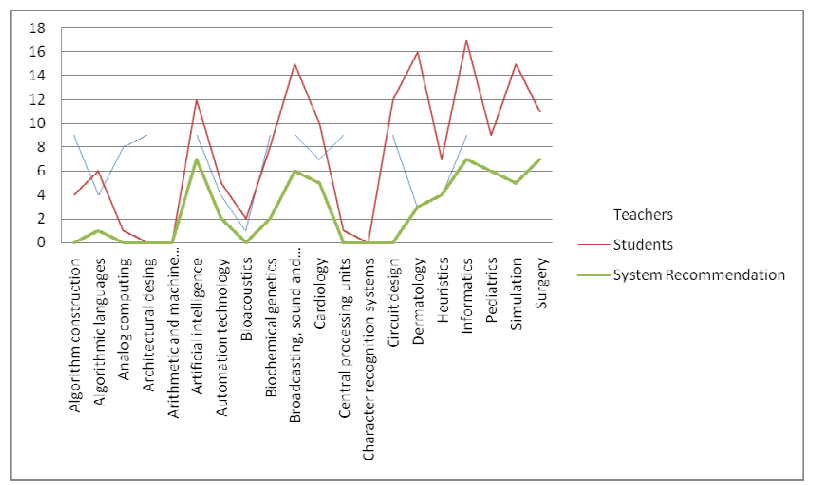

Fig. 3 Result of three recommendation system

\section{Conclusions and Future Work}

This first approach of integrating the two approximations (students and teachers) is promising have multi-agent system for recommending LO for students and teachers, operating together, recognize the user's needs and making a tailored recommendation, particularly to support the teaching-learning process. The system facilitates the composition of large training courses for teachers, by composing smaller elements. In addition, the system supports the learning of students based on their profile. The proposed MAS improves what already exists in the review found no recommendation systems that perform the two processes, helping teachers to develop new teaching materials and educational resources recommendation to students.

Currently it has developed a prototype for validation; as future work is to extend both theoretically and practically the MAS, using the advantages of both models: use of user profiles and collaborative filtering with CBR. Also defines the thresholds to determine the qualifications for making the most effective recommendation according to the two integrated approaches.

\section{References}

1. Li, J.Z.: Quality, Evaluation and Recommendation for Learning Object. In: International Conference on Educational and Information Technology, no. Iceit, pp. 533-537 (2010)

2. Gil, B., García, F.: Un Sistema Multiagente de Recuperación de Objetos de Aprendizaje con Atributos de Contexto. In: ZOCO 2007/CAEPIA (2007)

3. Niemann, K., Scheffel, M., Friedrich, M., Kirschenmann, U., Schmitz, H., Wolpers, M.: Usage-based Object Similarity. Journal of Universal Computer Science 16(16), 2272-2290 (2010)

4. Wooldridge, M., Jennings, N.R., Kinny, D.: A methodology for agent-oriented analysis and design. In: Proceedings of the Third Annual Conference on Autonomous Agents, AGENTS 1999, vol. 27, pp. 69-76 (1999) 
5. Casali, Gerling, V., Deco, C., Bender, C.: Sistema inteligente para la recomendación de objetos de aprendizaje. Revista Generación Digital 9(1), 88-95 (2011)

6. De la Prieta, F., Gil, A., Rodríguez, S., Martín, B.: BRENHET2, A MAS to Facilitate the Reutilization of LOs through Federated Search. In: Corchado, J.M., Pérez, J.B., Hallenborg, K., Golinska, P., Corchuelo, R. (eds.) Trends in Practical Applications of Agents and Multiagent Systems. AISC, vol. 90, pp. 177-184. Springer, Heidelberg (2011)

7. Rodríguez, P., Tabares, V., Duque, N., Ovalle, D., Vicari, R.M.: Multi-agent Model for Searching, Recovering, Recommendation and Evaluation of Learning Objects from Repository Federations. In: Pavón, J., Duque-Méndez, N.D., Fuentes-Fernández, R. (eds.) IBERAMIA 2012. LNCS, vol. 7637, pp. 631-640. Springer, Heidelberg (2012)

8. Wiley, D.A.: Connecting learning objects to instructional design theory: A definition, a metaphor, and a taxonomy. Learning Technology 2830(435), 1-35

9. Learning Technology Standards Committee, IEEE Standard for Learning Object Metadata. Institute of Electrical and Electronics Engineers, New York (2002)

10. JORUM, JISC Online Repository for [learning and teaching] Materials (2004), http://resources.jorum.ac.uk/xmlui

11. van de Sompel, H., Chute, R.: The aDORe federation architecture: digital repositories at scale. International Journal 9, 83-100 (2008)

12. Chesani, F.: Recommmendation Systems. Corso di laurea in Ingegneria Informatica, $1-32(2002)$

13. Mizhquero, K.: Análisis , Diseño e Implementación de un Sistema Adaptivo de Recomendación de Información Basado en Mashups. Revista Tecnológica ESPOL (2009)

14. Sanjuán, O., Torres, E., Castán, H., Gonzalez, R., Pelayo, C., Rodriguez, L.: Viabilidad de la aplicación de Sistemas de Recomendación a entornos de e-learning. Universidad de Oviedo, España (2009)

15. Verbert, K., Ochoa, X., Derntl, M., Wolpers, M., Pardo, A., Duval, E.: Semi-automatic assembly of learning resources. Computers \& Education 59(4), 1257-1272 (2012)

16. De, F., Pintado, P., Corchado, M.: Recuperación y Catalogación de Recursos Educativos mediante Organizaciones Virtuales y Filtrado Colaborativo 1 Introducción y objetivos (2011)

17. Jennings, N.R.: On agent-based software engineering. Artificial Intelligence 117(2), 277-296 (2000)

18. Boella, G., Hulstijn, J., Der Torre, V.: Virtual organizations as normative multiagent systems. In: HICSS. IEEE Computer Society (2005)

19. Huang, Y.-M., Chen, J.-N., Huang, T.-C., Jeng, Y.-L., Kuo, Y.-H.: Standardized course generation process using Dynamic Fuzzy Petri Nets. Expert Systems with Applications 34(1), 72-86 (2008)

20. Campos, R., Comarella, R., Azambuja, R.: Model of Recommendation System for for Indexing and Retrieving the Learning Object based on Multiagent System. In: MASLE $2012(2011)$

21. Li, M., Ogata, H., Hou, B., Uosaki, N., Yano, Y.: Personalization in Context-aware Ubiquitous Learning-Log System. In: 2012 IEEE Seventh International Conference on Wireless, Mobile and Ubiquitous Technology in Education, pp. 41-48 (March 2012) 